\title{
An examination by year of cases applied with caesarean hysterectomy because of placenta percreta in a tertiary centre: a retrospective cohort study
}

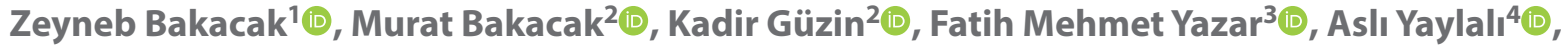 \\ Aytekin Uzkar² \\ ${ }^{1}$ Private Practice, Kahramanmaraş, Turkey \\ ${ }^{2}$ Department of Obstetrics and Gynecology, Kahramanmaraş Sütçü Imam University, School of Medicine, Turkey \\ ${ }^{3}$ Department of General Surgery, Kahramanmaraş Sütçü Imam University, School of Medicine, Turkey \\ ${ }^{4}$ Department of Histology and Embriyology, Kahramanmaraş Sütçü Imam University, School of Medicine, Turkey
}

\begin{abstract}
Objectives: To examine cases applied with caesarean hysterectomy because of placenta percreta by comparing changes in treatment strategies and complications according to year.

Material and methods: A retrospective examination was made of 93 patients applied with caesarean hysterectomy with a diagnosis of placenta percreta in 5-year periods of 2005-2009, 2010-2014, and 2015-2019. Demographic characteristics were recorded, and previous caesareans, history of myomectomy and curettage, gestational weeks, and infant birthweight. Intraoperative and postoperative findings were recorded as operating time, length of stay in hospital and Intensive Care Unit (ICU), transfusion requirement, the amount of erythrocyte suspension (ES) and fresh frozen plasma (FFP) transfused, and requirement for massive transfusion. Anaesthesia type, complications, and the preferred skin-uterus incision were also recorded.

Results: The 93 patients comprised 8 cases in the period 2005-2009, 23 in 2010-2014, and 62 in 2015-2019. The number of previous caesarean procedures was observed to increase in parallel with these case numbers. A significant increase was observed in the gestational week of birth, and infant birthweight, and a decrease in operating times. In later years there was seen to be a lower amount of ES and FFP transfused and fewer patients with massive transfusion. Preoperative diagnosis of placenta percreta, the highest preference for general anaesthesia, selection of midline vertical skin incision and uterine fundal incision were greatest in the period 2015-2019.

Conclusions: In cases with placenta percreta, of which there is an increasing incidence, maternal and infant outcomes can be optimised with prenatal diagnosis and planned caesarean hysterectomy by a multidisciplinary team with optimal prenatal preparation.
\end{abstract}

Key words: placenta percreta; cesarean hysterectomy; prenatal diagnosis; perinatal outcome

Ginekologia Polska 2021; 92, 4: 284-288

\section{INTRODUCTION}

Placenta accreta spectrum (PAS) describes a disease spectrum in which there is abnormal adherence of trophoblasts to the placenta. In $79 \%$ of cases the most mild form of placenta accreta is seen, in $14 \%$, placenta increta, and in $7 \%$, placenta percreta is formed with the most morbidity and mortality. While there is superficial myometrial invasion in placenta accreta, there is deep myometrial invasion in placenta increta, and in cases of placenta percreta, the myometrial invasion reaching the uterus serosa reaches as far as surrounding tissue and organs. The greatest clinical problem is massive bleeding when attempting to remove the placenta from the uterus after delivery of the fetus [1, 2]. Even if the procedure is switched to hysterectomy, this intense bleeding can lead to multi-organ failure disseminated intrvascular coagulation and even death. Surviving cases, especially those with complications related to urinary system damage, require ICU admittance and transfusion-related complications may be seen [2,3].

PAS occurs due to placental trophoblasts not attaching to scarred myometrium in the absence of a decidua basalis in a normal structure to which they would adhere secondary to endometrium damage [4]. The most common reasons for this endometrium damage are uterine surgery such as primarily caesarean procedures and myomectomy, and endometrial ablation applied by the cervical route, curettage and uterine artery embolisation [5]. 
As more caesarean deliveries are performed, there has been a rapid increase over theyears in the incidence of PAS. The incidence was first reported in 1937 by Irving and Hertig as 1 in 30,000 births, and this rate has now risen to 1 in 533 births $[6,7]$.

Significant changes have occurred in the 50 -year process of the diagnosis and management of PAS cases. A fundamental change came in diagnosis of the disease with the use of ultrasonography in the preoperative period in the 1980s [8-10]. Just as preoperative diagnosis allows the referral of patients to specialised centres and the opportunity to be operated on under elective conditions, it is also useful in respect of obtaining the opinions of assistive branches such as interventional radiology, general surgery and urology. Moreover, identification of the disease in the preoperative period allows several surgical modifications to be made, ranging from modifications to the skin and uterus incisions to uterus-sparing procedures $[1,9,10]$.

\section{Objectives}

The aim of this study was to examine cases applied with caesarean hysterectomy because of placenta percreta in our tertiary level clinic in the last 15 years by comparing changes in treatment strategies and complications according to year, and discussing these in the light of the relevant literature.

\section{MATHERIAL AND METHODS}

A retrospective examination was made of 125 patients applied with peripartum hysterectomy because of PAS in our university clinic between January 2005 and December 2019. From these cases, the study included 93 patients who were $>24$ gestational weeks with the diagnosis of placenta percreta confirmed histopathologically, and with all data available. This study conforms with the principles of the 2008 Declaration of Helsinki and was approved by the Local Ethics Committee of our university.

A record was made for each case of demographic characteristics, previous caesareans, history of myomectomy and curettage, gestational weeks, and infant birthweight. Intraoperative and postoperative findings were recorded as operating time, rates and length of stay in the Intensive Care Unit (ICU), total length of stay in hospital, the need for transfusion, the amount of erythrocyte suspension (ES) and fresh frozen plasma (FFP) transfused, and rates of the requirement for massive transfusion. The type of anesthesia, complications of damage to adjacent organs, and the preferred skin-uterus incision were also recorded. To investigate the change in approaches and outcomes of the patients, all the cases were separated into 5-year periods of 2005-2009, 2010-2014, and 2015-2019.

\section{Statistical Analysis}

Data obtained in the study were analysed statistically using the Statistical Package for the Social Sciences vn.
21.0 software (SPSS, Armonk, New York, IL, USA). Conformity of continuous data to normal distribution was assessed with the Kolmogorov-Smirnov test. Quantitative variables were stated as mean \pm standard deviation (SD) and median range (minimum-maximum) values. Multiple group comparisons were made with ANOVA and the Tukey HSD test. Categorical data were stated as number (n) and percentage (\%). A value of $\mathrm{p}<0.05$ was accepted as statistically significant.

\section{RESULTS}

The 93 patients comprised 8 cases in the period 20052009, 23 cases in 2010-2014, and 62 cases in 2015-2019. The increase in the number of cases was determined to be statistically significant $(p<0.004)$ (Fig. 1).

No statistically significant difference was determined between the groups in respect of patient age, BMl, gravida, parity, and history of myomectomy and curettage ( $p>0.05$ for all) (Tab. 1). The number of previous caesarean procedures was mean $1.74 \pm 1.00$ for the period of 2005-2009, $2.15 \pm 0.95$ for 2010-2014, and 2.79 \pm 1.03 for 2015-2019, and the increase was determined to be statistically significant $(p=0.012)$.

In the examination of the intra-operative and postoperative findings, there was observed to be a statistically significant increase in the gestational week at which patients gave birth and a parallel increase in infant birthweight $(p=0.026$, $p=0.035$ ). When the operating times were compared, there was seen to be a statistically significant decrease in the times over the years $(p=0.004)$. No statistically significant difference was observed between the groups in respect of the rates of adult ICU admittance and the length of stay in ICU $(p=0.157, p=0.519)$. The total length of hospital stay was found to be statistically significantly shorter in the period 2015-2019 compared to the earlier years $(p=0.026)$. No statistically significant difference was seen according to the years in respect of patients requiring transfusion and hypogastric artery ligation $(p=0.215, p=0.349$, respectively). As the years progressed there was determined to

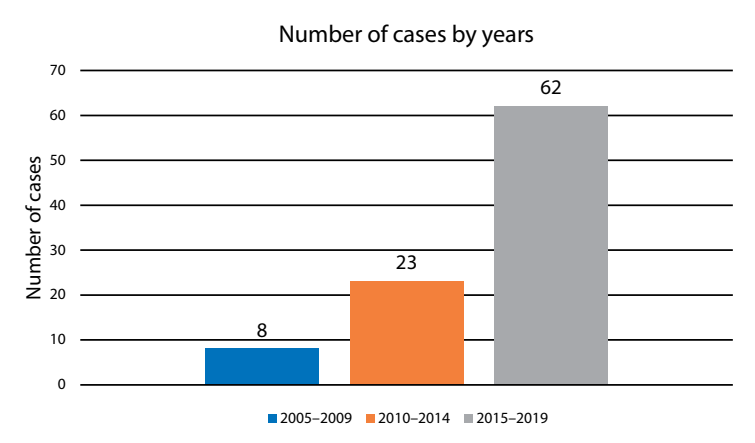

Figure 1. Number of cases by years 
Table 1. Demographic characteristics of the cases

\begin{tabular}{|l|l|l|l|l|}
\hline & $\mathbf{2 0 0 5 - 2 0 0 9}(\mathbf{n}=\mathbf{8})$ & $\mathbf{2 0 1 0 - 2 0 1 4}(\mathbf{n = 2 3 )}$ & $\mathbf{2 0 1 5 - 2 0 1 9}(\mathbf{n}=\mathbf{6 2})$ & p value \\
\hline Age [years] & $33.20 \pm 4.54$ & $32.69 \pm 3.62$ & $34.24 \pm 4.31$ & 0.303 \\
\hline BMI $\left[\mathrm{kg} / \mathrm{m}^{2}\right]$ & $25.87 \pm 2.30$ & $26.34 \pm 2.72$ & $26.47 \pm 3.29$ & 0.468 \\
\hline Gravida & $4.33 \pm 1.86$ & $4.40 \pm 1.81$ & $4.64 \pm 0.99$ & 0.756 \\
\hline Parity & $3.40 \pm 0.89$ & $2.50 \pm 1.29$ & $2.85 \pm 0.78$ & 0.245 \\
\hline $\begin{array}{l}\text { Number of previous caesarean procedures } \\
\text { History of myomectomy }\end{array}$ & $1.74 \pm 1.00$ & $2.15 \pm 0.95$ & $2.79 \pm 1.03$ & $\mathbf{0 . 0 1 2}$ \\
\hline History of curettage & $0 / 8(0 \%)$ & $1 / 23(0.043 \%)$ & $3 / 62(0.048 \%)$ & 0.521 \\
\hline
\end{tabular}

Data are stated as mean \pm standard deviation or number $(\mathrm{n})$ and percentage (\%). Significant $\mathrm{p}$ values are shown in bold and italics; BMI — body mass Index

Table 2. Intra-operative and postoperative findings of the cases

\begin{tabular}{|l|l|l|l|l|}
\hline & $\mathbf{2 0 0 5 - 2 0 0 9}(\mathbf{n = 8})$ & $\mathbf{2 0 1 0 - 2 0 1 4}(\mathbf{n}=\mathbf{2 3})$ & $\mathbf{2 0 1 5 - 2 0 1 9}(\mathbf{n = 6 2})$ & $\mathbf{p ~ v a l u e ~}$ \\
\hline $\begin{array}{l}\text { Gestational week } \\
\text { Infant birthweight [g] }\end{array}$ & $30.20 \pm 6.61$ & $34.21 \pm 4.03$ & $35.34 \pm 4.80$ & 0.026 \\
\hline Operating time [mins] & $2247.50 \pm 376.29$ & $2508.75 \pm 826.24$ & $2794.14 \pm 944.24$ & 0.035 \\
\hline Length of stay in adult ICU [days] & $128.05 \pm 36.48$ & $110.54 \pm 32.41$ & $89.66 \pm 27.23$ & 0.004 \\
\hline Total length of hospital stay [days] & $2.38 \pm 2.14$ & $2.50 \pm 1.54$ & $2.72 \pm 2.02$ & 0.519 \\
\hline Transfusion requirement (n/\%) & $6.53 \pm 3.12$ & $5.02 \pm 2.56$ & $4.54 \pm 1.43$ & 0.026 \\
\hline Transfused ES [units] & $7(87.5 \%)$ & $20(86.9 \%)$ & $50(80.6 \%)$ & 0.215 \\
\hline Transfused FFP [units] & $6.52 \pm 2.94$ & $5.94 \pm 1.82$ & $4.31 \pm 1.91$ & 0.039 \\
\hline Massive transfusion requirement (n/\%) & $3.36 \pm 2.12$ & $2.00 \pm 1.30$ & $2.09 \pm 0.83$ & 0.041 \\
\hline Hypogastric artery ligation (n/\%) & $6(87.5 \%)$ & $17(73.9 \%)$ & $19(30.6 \%)$ & 0.016 \\
\hline Dato & $2(25 \%)$ & $5(21.7 \%)$ & $12(19.6 \%)$ & 0.349
\end{tabular}

Data are stated as mean \pm standard deviation or number $(\mathrm{n})$ and percentage (\%). Significant $\mathrm{p}$ values are shown in bold and italics; ICU — intensive care unit; ES - erythrocyte suspension; FFP — fresh frozen plasma

be a statistically significantly lower amount of ES and FFP transfused and fewer patients applied with massive transfusion ( $p=0.039, p=0.041, p=0.016$, respectively) (Tab. 2).

When all the cases were examined, the rate of preoperative diagnosis of placenta percreta was determined to be statistically significantly highest in the period 2015$2019(p=0.001)$.

The preference for general anaesthesia was determined to be statistically significantly higher in 2015-2019 compared to the other periods $(p=0.021)$. No difference was seen between the 5 -year periods in respect of perioperative complications $(p=0.458)$. In the comparison of the change in skin and uterus incisions over the years, the preference of skin incision was observed to change from Pfannenstiel to midline vertical, and in parallel with this, there was a significant change in uterine incision from Kerr incision to fundal incision $(p=0.001)$ (Tab. 3).

\section{DISCUSSION}

For patients with a diagnosis of placenta percreta, caesarean hysterectomy is the current standard treatment, in which the infant is delivered far from the placenta (usually from the fundus) under elective conditions at an appropriate gestational week before the onset of labour and bleeding, and the uterine incision is closed without touching the placenta [11]. Cases with a confirmed preoperative diagnosis of placenta percreta in our clinic are applied with caesarean hysterectomy as standard.

In recent years, there has been an increasing trend in cases applied with caesarean hysterectomy because of placenta percreta in our clinic, and throughout the world [12]. The most common risk factor for placenta accreta is a history of caesarean delivery. A systematic review showed that this rate reached $50-67 \%$ in patients with placenta previa and a history of at least 3 caesarean deliveries [13]. In the current series, there was a noticeable increase in the history of caesarean deliveries according to the years. The increasing rate of caesareans throughout the world in the last few decades is the primary reason for the increase in the incidence of placenta percreta [1].

An interesting finding in the current series was that as the years progressed, there was an increase in the gestational week at which the infant was delivered and in parallel, an increase in infant birthweight. It was noteworthy that in the last 10 years, the cases were operated on at 34-35 weeks. When determining the optimal time of de- 


\begin{tabular}{|c|c|c|c|c|}
\hline & $2005-2009(n=8)$ & $2010-2014(n=23)$ & $2015-2019(n=62)$ & p value \\
\hline \multicolumn{4}{|c|}{ Prenatal Diagnosis of Placenta percreta } & 0.001 \\
\hline Yes & 1 & 8 & 55 & \\
\hline No & 7 & 15 & 7 & \\
\hline \multicolumn{4}{|l|}{ Type of Anaesthesia } & 0.021 \\
\hline General & 4 & 13 & 59 & \\
\hline Regional & 4 & 10 & 3 & \\
\hline \multicolumn{4}{|c|}{ Perioperative surgical complications } & 0.458 \\
\hline Bladder damage & 2 & 4 & 15 & \\
\hline Ureter damage & 0 & 1 & 1 & \\
\hline Intestine damage & 0 & 0 & 0 & \\
\hline \multicolumn{4}{|l|}{ Skin incision } & 0.013 \\
\hline Pfannenstiel & 8 & 10 & 10 & \\
\hline Midline vertical & 0 & 13 & 52 & \\
\hline \multicolumn{4}{|l|}{ Uterine incision } & 0.001 \\
\hline Kerr & 7 & 10 & 0 & \\
\hline Low vertical & 1 & 0 & 10 & \\
\hline High vertical & 0 & 3 & 2 & \\
\hline Fundal & 0 & 10 & 50 & \\
\hline
\end{tabular}

Data are stated as mean \pm standard deviation or number $(n)$ and percentage $(\%)$. Significant $p$ values are shown in bold and italics

livery for these elective cases, both maternal and infant well-being must be taken into consideration, but there is no consensus as yet on this subject. The infrastucture of the neonatal ICU (NICU) and experience with pre-term infants at the hospital where the birth is to take place must also be considered. The success rates of most NICUs with newborns born after 34 gestational weeks are high. There is known to be an increased risk of maternal bleeding after the 36th gestational week [14]. Therefore, the optimal birthweek is recommended by the American College of Obstetricians and Gynecologists (ACOG) as $34^{+0}-35^{6 / 7}$ [15] and by the Royal College of Obstetricians and Gynaecologists (RCOG) as $35^{+0}-36^{+6}[16]$.

Another interesting point of the current study was that lower amounts of blood products were transfused to patients in more recent years. In parallel, there was observed to be an increase in the rate of patients operated on with a preoperative diagnosis and shorter operating times. As the total amount of blood loss may be less in surgery under elective conditions compared to patients who start vaginal bleeding in the antenatal period and are operated on after losing blood from the vaginal route, there could therefore be less requirement for transfusion. However, another reason for shorter operating times and less bleeding could be that the operations of these types of cases under elective conditions are performed by more experienced surgeons. Therefore, a prenatal diagnosis is very important for these cases, so that surgery can be applied under elective conditions, and patients can be referred to centres where there are more experienced surgeons and a multidisciplinary approach is possible (obstetric surgeons, anaesthesiologists, neonatologists, interventional radiologists, blood bank and nursing personnel experienced in this subject) [17].

The preference of anaesthesia type for these cases was seen to have shifted over the years from regional anaesthesia towards general anaesthesia. This can be considered to be due to the increasing rate of diagnosis, because in caesarean cases where an unpredicted hysterectomy is made using a Pfannenstiel incision with no abnormal bleeding risk, the first choice is regional anaesthesia for reasons such as better postoperative pain relief, fewer adverse effects and more rapid recovery. However, in placenta percreta cases with a complex procedure applied with a large midline incision, where there is a greater possibility of more bleeding, hypovolemia and hemodynamic instabiity, the preferred anaesthesia method is general anaesthesia with a controlled airway [18]. In cases with suspected placenta percreta where a definitive diagnosis is needed intraoperatively, epidural anaesthesia can be applied as it can be later converted to general anaesthesia to be able to perform a hysterectomy.

The incision preferences were seen to have changed in recent years to a midline vertical abdominal incision and a fundal uterus incision. This is because in these patients diagnosed preoperatively with placenta percreta, the deci- 
sion to perform hysterectomy has been made definitely before the operation. Although there are case series showing good results obtained with transverse incisions such as Pfannenstiel, Maylard and Cherney [19], when performing a hysterectomy in a large pregnant patient, it can be considered necessary to use a midline vertical incision to provide sufficient visualisation and to easily reach the uterus and the retroperitoneal large vessels. Another advantage of midline vertical incision is that it provides easy access to the uterine fundus when the infant has to be delivered far from the part of the uterus where the placenta has settled and in most cases this region is the uterus fundal section [11].

No significant difference was observed between the cases in this series in respect of complication rates. The majority of intraoperative complications are urinary system complications, most of which are bladder perforations at an incidence of up to $17 \%$ [20]. When there is placental invasion of the bladder and in the presence of very dense bladder adhesions, bladder complications are inevitable. Prenatal diagnosis and differences in incision type and the surgeon's experience can be considered not to have changed the rates of bladder complications.

There were some limitations to this study, primarily that when analysing the cases they were examined in 3 groups of 5 -year periods. However, in the early years there were very few cases, and in more recent years there were many more cases. Therefore, to be able to statistically analyse the cases it was necessary to group them in this way. Another limitation could be considered to be that there were no follow-up data of the newborn infants of the cases.

\section{CONCLUSIONS}

There is an increasing incidence of placenta accreta, for which the primary risk factors are a history of caesarean delivery and placenta previa. A prenatal diagnosis can reduce morbidity and mortality. Ultrasound is the best imaging modality in prenatal diagnosis. The timing of the birth should be planned on an individual basis but is generally at 34-36 weeks. The standard treatment for placenta accreta is planned caesarean and hysterectomy. Maternal and infant outcomes can be optimised with a multidisciplinary team and optimal prenatal preparation.

\section{Conflict of interest}

The authors have no potential conflict of interests to declare.

\section{REFERENCES}

1. Silver RM, Branch DW. Placenta Accreta Spectrum. N Engl J Med. 2018; 378(16): 1529-1536, doi: 10.1056/NEJMcp1709324, indexed in Pubmed: 29669225.
2. Wu S, Kocherginsky M, Hibbard JU. Abnormal placentation: twenty-year analysis. Am J Obstet Gynecol. 2005; 192(5): 1458-1461, doi: 10.1016/j. ajog.2004.12.074, indexed in Pubmed: 15902137.

3. Bailit JL, Grobman WA, Rice MM, et al. Eunice Kennedy Shriver National Institute of Child Health and Human Development (NICHD) Maternal-Fetal Medicine Units (MFMU) Network. Morbidly adherent placenta treatments and outcomes. Obstet Gynecol. 2015; 125(3): 683-689, doi: 10.1097/AOG.0000000000000680, indexed in Pubmed: 25730233.

4. Silver RM, Clark EAS, Silver RM, et al. National Institute of Child Health and Human Development Maternal-Fetal Medicine Units Network. Maternal morbidity associated with multiple repeat cesarean deliveries. Obstet Gynecol. 2006; 107(6): 1226-1232, doi: 10.1097/01. AOG.0000219750.79480.84, indexed in Pubmed: 16738145.

5. Miller DA, Chollet JA, Goodwin TM. Clinical risk factors for placenta previa-placenta accreta. Am J Obstet Gynecol. 1997; 177(1): 210-214, doi: 10.1016/s0002-9378(97)70463-0, indexed in Pubmed: 9240608.

6. Baldwin HJ, Patterson JA, Nippita TA, et al. Antecedents of Abnormally Invasive Placenta in Primiparous Women: Risk Associated With Gynecologic Procedures. Obstet Gynecol. 2018; 131(2): 227-233, doi: 10.1097/AOG.0000000000002434, indexed in Pubmed: 29324602.

7. Irving C. and Hertig AT. A study of placenta accreta. Surg Gynec Obst. 1937; 64: 178-200.

8. Tabsh KM, Brinkman CR. 3rd, King W. Ultrasound diagnosis of placenta increta. J Clin Ultrasound. 1982; 10(6): 288-290.

9. Allen L, Jauniaux E, Hobson S, et al. FIGO Placenta Accreta Diagnosis and Management Expert Consensus Panel. FIGO consensus guidelines on placenta accreta spectrum disorders: Nonconservative surgical management. Int J Gynaecol Obstet. 2018; 140(3): 281-290, doi: 10.1002/ijgo.12409, indexed in Pubmed: 29405317.

10. Eller AG, Porter TF, Soisson $P$, et al. Optimal management strategies for placenta accreta. BJOG. 2009; 116(5): 648-654, doi: 10.1111/j.1471-052 8.2008.02037.x, indexed in Pubmed: 19191778.

11. Di Mascio D, Calì G, D'antonio F. Updates on the management of placenta accreta spectrum. Minerva Ginecol. 2019; 71(2): 113-120, doi: 10.23736/S0026-4784.18.04333-2, indexed in Pubmed: 30486635.

12. Mogos MF, Salemi JL, Ashley M, et al. Recent trends in placenta accreta in the United States and its impact on maternal-fetal morbidity and healthcare-associated costs, 1998-2011. J Matern Fetal Neonatal Med. 2016; 29(7): 1077-1082, doi: 10.3109/14767058.2015.1034103, indexed in Pubmed: 25897639.

13. Klar M, Michels KB. Cesarean section and placental disorders in subsequent pregnancies--a meta-analysis. J Perinat Med. 2014; 42(5): 571-583, doi: 10.1515/.jpm-2013-0199, indexed in Pubmed: 24566357.

14. Robinson BK, Grobman WA. Effectiveness of timing strategies for delivery of individuals with placenta previa and accreta. Obstet Gynecol. 2010; 116(4): 835-842, doi: 10.1097/AOG.0b013e3181f3588d, indexed in Pubmed: 20859146.

15. American College of Obstetricians and Gynecologists, Society for Maternal-Fetal Medicine. Obstetric Care Consensus No. 7: Placenta Accreta Spectrum. Obstet Gynecol. 2018; 132(6): e259-e275, doi: 10.1097/AOG.0000000000002983, indexed in Pubmed: 30461695.

16. Jauniaux E, Alfirevic Z, Bhide AG, et al. Royal College of Obstetricians and Gynaecologists. Placenta Praevia and Placenta Accreta: Diagnosis and Management: Green-top Guideline No. 27a. BJOG. 2019; 126(1): e1-e48, doi: 10.1111/1471-0528.15306, indexed in Pubmed: 30260097.

17. Eller AG, Bennett MA, Sharshiner M, et al. Maternal morbidity in cases of placenta accreta managed by a multidisciplinary care team compared with standard obstetric care. Obstet Gynecol. 2011;117(2 Pt 1):331-337, doi: 10.1097/aog.0b013e3182051db2, indexed in Pubmed: 21309195.

18. Lilker SJ, Meyer RA, Downey KN, et al. Anesthetic considerations for placenta accreta. Int J Obstet Anesth. 2011; 20(4): 288-292, doi: 10.1016/j. ijoa.2011.06.001, indexed in Pubmed: 21840207.

19. Collins SL, Alemdar B, van Beekhuizen $\mathrm{HJ}$, et al. International Society for Abnormally Invasive Placenta (IS-AIP). Evidence-based guidelines for the management of abnormally invasive placenta: recommendations from the International Society for Abnormally Invasive Placenta. Am J Obstet Gynecol. 2019; 220(6):511-526, doi: 10.1016/j.ajog.2019.02.054, indexed in Pubmed: 30849356.

20. Clausen C, Lönn L, Langhoff-Roos J. Management of placenta percreta: a review of published cases. Acta Obstet Gynecol Scand. 2014; 93(2): 138-143, doi: 10.1111/aogs.12295, indexed in Pubmed: 24266548. 